\title{
Editorial
}

\section{Linke Ortsbestimmungen der Gegenwart}

Das sozialistische Lager und die Sowjetunion existieren nicht mehr. Die Koordinaten der politischen Landschaft und die Angelpunkte linker Theorie und Strategie haben sich daher verschoben. Es fragt sich natürlich: wohin? Diese Frage stellt sich weniger orthodoxen Parteiintellektuellen - die ohnehin nicht auf Fragen, sondern auf vorgefertigte Antworten spezialisiert waren. Sie stellt sich vielmehr jener $»$ Neuen Linken«, die seit den 60er Jahren in Opposition gegen den Sozialismus sowjetischen Typs entstanden war, und nun bemerkt, daß sie mit dem gemeinsamen Gegenstand dieser Opposition ihren gemeinsamen Nenner verloren hat. Sie stellt sich schließlich all denen, die von den triumphalistischen Wortführern des Wirtschaftsliberalismus erstaunt vernehmen, daß nicht nur der Staatssozialismus, sondern auch die sozialstaatliche »Institutionalisierung des Klassenkonflikts« ausgespielt habe.

Von einer Krise des Marxismus im Jahre $1993 \mathrm{zu}$ sprechen wie noch Ende des vergangenen Jahrhunderts während des $\gg$ Revisionismusstreits « oder Anfang der $30 \mathrm{er}$ bei der Her- aufkunft des Faschismus und Nationalsozialismus und dann wieder Mitte der 70er Jahre nach dem abgebrochenen Aufbruch von ' 68 wäre eine Untertreibung. Die sozialistischen Projekte, die in der Tradition der II., III. und IV. Internationale erarbeitet worden sind, sind keine $\mathrm{Al}-$ ternativen zur kapitalistischen Form der Vergesellschaftung. Kann also auch die Theorie ad acta historiae gelegt werden? Nach dem Scheitern des einzigen historisch real-sozialistischen Projekts werden diese Projekte in eine Grabkammer gebettet. Darüber wird eine post-sozialistische Pyramide post-moderner Theorie und liberaler Politikfragmente errichtet. Über »die Linke«, über Sozialismus heute nachzudenken, ist nur möglich, wenn man sich von alten Dogmen verabschiedet. Dabei muß man sich allerdings nicht kleinlaut in eine windstille Ecke verkrümeln, um der Zugluft der Geschichte zu entgehen. Denn »die Linke« gab es auch vor 1917 .

Warum soll es sie nach der mehr als 70 Jahre währenden Geschichte der »verratenen Revolution « nicht immer noch und immer wieder geben? Niemand anderes als Max Weber 
rechnete den »modernen Sozialismus« dem »modernen Gegensatz zwischen großindustriellem Unternehmer und freiem Lohnarbeiter « als komplementäre Erscheinung zu. Die säkularisierte, moderne kapitalistische Gesellschaft ist weder perfekt noch sakrosankt. Also ist sie für verschiedene Projekte und Entwikklungspfade offen, auch für linke Ideen, Programme und Bewegungen. »Kapitalismus «, das heißt nicht nur demokratische Bürgergesellschaft, Umgangsformen in persönlicher Freiheit, Markt und ökonomische Effizienz - Normen und praktizierte Lebensformen, auf die sich zu verständigen niemandem schwer fallen dürfte. Kapitalismus steht auch für Ausbeutung, für ungleiche Lebenschancen, für Krisen, für Abhängigkeit und Fremdbestimmung und für globalisierte Naturzerstörung. Herausforderungen für Kritik verbleiben also auch am sogenannten »Ende der Geschichte«, das sich nur wenige Jahre nach dem Ende der West-Ost-Konfrontation als eine unüberschaubare Gemengelage von Konflikten, Kriegen und Interventionen dem Zeitgenossen darbietet.

Doch wie soll diesen Herausforderungen begegnet werden, nachdem viele Gewißheiten entschwunden sind? Die sich überschlagenden Ereignisse in Osteuropa, in der Sowjetunion und anderswo sind Trümmerhaufen auf einem historischen Pfad, der sich in unwegsamen Gelände verliert.

\section{Fellow Traveller und Dissidenten}

Die Linke in der westlichen Welt, gleichgültig wie sie sich theoretisch, ideologisch und politisch definierte, war im Ost-West-Gegensatz lokalisiert. Der Linken im Osten wurde ein Maulkorb umgehängt und sie mußte sich zweideutig verhalten. In Opposition zum Regime schien sie ohne ihr weiteres Zutun auf die Seite des Blockgegners zu geraten; im Streben nach Emanzipation begab sie sich jedoch in ein Niemandsland jenseits der Blöcke, wo Kontaktaufnahme und Verständigung mit der ebenfalls »heimatlosen« westlichen Linken nur selten gelang. Für die Ausgrenzung der Linken aus dem je eigenen System sorgten im Westen der vergleichsweise harmlose Fellow-Traveller-Vorwurf oder die Empfehlung, doch »nach drüben« zugehen. Im Osten wurde diese Empfehlung mit Ausbürgerung, Arrest und Isolation der »Dissidenten « brutal in die Tat umgesetzt. Die Logik des Kalten Kriegs folgte dem binären Code des Dafür- oder DagegenSeins: tertium non datur. Darin waren sich McCarthy und das Moskauer ZK einig. Die Versuche, unabhängige Positionen zu beziehen, waren riskant und obendrein auch folgenlos. Denn die Linken, immer in Opposition zum jeweiligen Regime, waren auch vom Blockgegner nicht einfach zu goutieren, entzogen sie sich doch dem binären Schema. Die östlichen Linken paßten nicht in die westliche Politik der Blockstabi- 
lisierung; die Linken im Westen waren den östlichen Machthabern immer suspekt, weil sie das Monopol des von Breschnew als »real-existierend « definierten Sozialismus in Frage stellten. Jene, die in der bipolaren Welt zwischen 1947 und 1985 einen Weg zwischen den oder jenseits der Blöcke suchten, hatten kein leichtes Spiel, auch wenn sie die Sympathien der (unabhängigen) Linken auf sich vereinen konnten, wie Titos Jugoslawien in den frühen $50 \mathrm{er}$ oder Castros Kuba in den 60er Jahren, Dubceks Tschechoslowakei im Jahre 1968 oder zehn Jahre später das sandinistische Nicaragua.

Doch gerade weil der als Realität beschworene Sozialismus aus der Manege gejagt worden ist, sind der Linken nun neue Aufgaben und Chancen im Welttheater des »PostKommunismus « zugewachsen. Befreit vom ständigen Abgrenzungszwang - daß eine westliche Linke »unabhängig «, »neu«, »undogmatisch «, »anti-autoritär « und »nichtorthodox « sein müsse, um nicht mit dem Marxismus-Leninismus und der Politik des Sowjetblocks identifiziert zu werden - kann sie ihre Kritik auf den als alternativlos präsentierten Kapitalismus konzentrieren. Kritik setzt allerdings Theorie voraus und eine Vision, aus der sie ihre Kraft zieht.

Die Marx'sche Kritik der politischen Ökonomie nährte sich aus der Utopie einer nachkapitalistischen Gesellschaft, wobei Marx im szientifischen 19. Jahrhundert der Wissen- schaft (dem $»$ wissenschaftlichen Sozialismus «) die Aufgabe der Kritik der kapitalistischen Produktionsweise anvertraute. Heute kann sich eine solche Kritik nicht mehr naiv auf »positive Wissenschaft" berufen. Angesichts der überwältigenden »Positivität« des globalisierten Kapitalismus und der ihm zur Verfügung stehenden Wissenschaft, kommt es mehr denn je darauf an, den »Möglichkeitssinn « zu schärfen. Jede Wirklichkeit enthält, wie Robert Musil in diesem Sinn bemerkte, auch eine mögliche Wirklichkeit: »...darin, daß eine Möglichkeit nicht Wirklichkeit ist, drückt sich nichts anderes aus, als daß die Umstände, mit denen sie gegenwärtig verflochten ist, sie daran hindern, denn andernfalls wäre sie ja nur eine Unmöglichkeit; löst man sie nun aus ihrer Bindung und gewährt ihr Entwicklung, so entsteht die Utopie...«. Wer über die Wirklichkeit redet, sollte also den versteckten Möglichkeiten von Befreiung nachspüren: aus den vielfältigen Umgarnungen der Konsumwelt, aus den das menschliche Maß übersteigenden Zeitansprüchen, den Leistungs- und Konkurrenzzwängen, der zunehmenden Verelendung eines großen Teils der Bevölkerung in der Dritten Welt und nicht zuletzt aus einem die Tragfähigkeit der globalen Ökosysteme weit überfordernden Naturverbrauch. 


\section{Gespenster}

75 Jahre nach der russischen Revolution folgte die sowjetische Implosion - was kommt danach? Wie wird die Übergangsperiode, in die alle osteuropäischen Länder eingetreten sind, ausgehen? Kann der 1917 zerschnittene rote Faden der Geschichte von 1789 einfach wieder aufgegriffen werden, so wie es die These von der »nachholenden Revolution « nahelegt? Findet Ariadne so leicht aus dem Labyrinth der Geschichte, oder ist der Faden, an dem sich Brüder (und Schwestern) zur Sonne, zur Freiheit emporhangeln können, heillos verknotet? Ist es möglich, daß sich Artikulationsmuster zwischen dem Alten, dem Vorrevolutionären, dem was in der Zwischenzeit geschehen und entstanden ist, und dem kapitalistischen Weltgeschehen unserer Tage herausbilden? Welche Gesellschaften entstehen im Zuge der Transformationsprozesse, welche Zerfallsprodukte bleiben als Residuen auch in der neuen Zeit und wie lange?

Der alte proletarische Internationalismus - selbst noch abgewandelte Form des Universalismus der französischen Revolution und ideologischer Hintergrund der Ausdehnung des Sozialismus sowjetischen Typs über die russischen Grenzen (auch die französische Revolution war freilich expansiv) - scheint angeblich »wiedergeborenen « Formen von Nationalismus Platz zu machen; Reliquien aus der vorrevolutionären
Epoche werden poliert. Ausgemusterte Zuschreibungen von kollektiven Identitäten sind brandaktuell. Das mit dem Kapital und Lenins Schriften gerüstete »Gespenst des Kommunismus « ging bis zum Ende des »kurzen 20. Jahrhunderts« in Europa um. Ihm folgt das waffenstarrende und mordbrennende Gespenst des rassistischen Nationalismus. In den Triumph von Marktwirtschaft und Demokratie mischen sich schon vor Ende der Feier die Tschetniks und Ustaschas, johlende Neonazis und Skinheads.

Die Entstehung vieler neuer Nationen in Osteuropa ist daher alles andere als ein produktiver Prozeß, es ist die Herausbildung eines »entzivilisierten Kapitalismus (vgl. PROKLA 85), das Insignium der Vergesellschaftungsdefizite des Marktes (wie übrigens nicht nur in Osteuropa, sondern in vielen anderen Weltgegenden auch; der reiche, »zivilisierte« Westen nicht ausgenommen). Jugoslawien ist dafür ein unendlich trauriges Beispiel, das im Kaukasus, vielleicht im Baltikum oder in Rußland Schule machen könnte - freilich mit dem unangenehmen Unterschied, daß die dortigen Kombattanten nicht nur über Kalaschnikows, sondern auch über Atomwaffen verfügen. Daß auch im Westen Europas Nationen, Regionen oder Ethnien nach Unabhängigkeit streben, ist natürlich kein Trost. Soll für Basken, Korsen, Walliser etc. nicht gelten, was für die Balten, Moldavier oder Georgier für selbst- 
verständlich gehalten wird? Wer politische Einheiten auf nationale oder ethnische Unterscheidungen gründen will, wird die Landkarte Europas und der Welt permanent neu zeichnen müssen. Staaten lösen sich auf, bilden sich neu, vereinigen und spalten sich - ohne Konflikt und Krieg freilich wird dies alles nicht abgehen. Linke Kokettiererei mit der "nationalen Frage « hebt die Sargdeckel, aus denen die glatzköpfigen Vampire zum Mitternachts$\tan z$ entsteigen.

\section{Fundamentalismus}

Gerade die Krise der kapitalistischen Weltwirtschaft seit Mitte der 70er Jahre hat deutlich gemacht, wie sehr in einer Phase des Niedergangs und der Destruktion technische, arbeitsorganisatorische und ideologische Innovationen entwickelt und implementiert werden können. Kein Wunder, daß die Schumpetersche These von der »schöpferischen Zerstörung « in der gegenwärtigen Krise wieder Hochkonjunktur erlebt, bisweilen als zynische Feststellung, manchmal als Trostrede. In der Krise der 90er Jahre jedenfalls sind Strukturveränderungen am Werk, die wohl diejenigen der letzten GroBen Krise noch in den Schatten stellen. Das Niveau der Arbeitslosigkeit dürfte auch bei einem erneuten Aufschwung in den Industrieländern des Westens deutlich höher als in den 80er Jahren liegen, die Landkarte der Industriestandorte wird weltweit neu gezeichnet. Für die Transformationsgesellschaften Osteuropas wäre es sicherlich leichter, sich in einer Boomperiode an den Weltmarkt anzukoppeln. »Blühende Landschaften« wären eher im Aufschwung als in der Krise zu kultivieren. Die versprochenen und erwarteten Gratifikationen der »samtenen Revolution « kommen so allenfalls mit Zeitverzögerung, wenn überhaupt, zustande. Im Westen dagegen steigt mit der Ungewißheit über die Zukunft auch die Unsicherheit und in ihr der Ruf nach Sicherheit, nach Absicherung, beispielsweise der Grenzen gegen die von außen kommenden Zumutungen. Das ist eine große Zeit für Populismen und Fundamentalismen, also für Abgrenzungen einerseits und die Herstellung neuer prekärer Identitäten, die auf Vergangenes rekurrieren, andererseits. Am »Ende der Geschichte « ist dieses Vergangene von der »tabula rasa « gefegt worden, nun wird es mangels produktiver Perspektiven erneut aufgetischt.

Gegen die neuen und irritierenden Fundamentalismen wird auch die Linke in eine neu errichtete Front einzugemeinden versucht. Die dramatis personae der traumatischen Systemauseinandersetzung in diesem Jahrhundert, ehemalige Kommunisten und Antikommunisten, werden in der FAZ-Serie "What's Left? « aufgerufen, die Erbschaft der Aufklärung gegen den bedrohlich heraufziehenden Irrationalismus der Anti-Aufklärung und gegen den 
Fundamentalismus der Religion, der Ethnien, der politischen Irrationalismen gemeinsam $\mathrm{zu}$ verteidigen. Die alte Front am Eisernen Vorhang ist zusammengebrochen und schon soll ein neuer Kreuzzug organisiert werden. Offenbar wird ein beträchtlicher Teil der Menschheit des Kollapses des realen Sozialismus, des »Siegs im Kalten Krieg « und der Errichtung der neuen Weltordnung mit politisch-parlamentarischer Demokratie und freiem Markt nicht so recht froh. Das ist verständlich, denn die Gratifikationen des westlichen Zivilisationsmodells sind extrem ungleich auf dem Globus verteilt. Den einen das Auto, ein voller Kühlschrank und der grüne Punkt, den anderen die Giftfässer und der Wohlstandsmüll, um, wie es aus der Weltbank heißt, dem Zustand von $\gg$ underdevelopment $\ll$ und »underpollution « ein Ende zu bereiten.

An dieser Stelle offenbart sich eine fundamentale Inkonsequenz liberalen Denkens. Die Emphase, mit der beispielsweise Ralf Dahrendorf die Weltbürgerschaft gegen falsche Ersatzutopien begründet und das $\gg$ Projekt der Moderne« verteidigt, das verbissene Pochen auf der Nicht-Revozierbarkeit von individueller Freiheit, Differenz, Distanz, von ziviler Gesellschaft und freiem Markt, müssen darauf befragt werden, ob diese Prinzipien überhaupt auf einem kapitalistisch dominierten Globus mit mehr als sechs Milliarden Menschen am Ende des zweiten Jahrtausend verallgemeinerbar sind.
Kant konnte noch von Weltbürgerschaft als einem emanzipatorischen Prinzip ausgehen. Denn im Königsberg seiner Zeit waren Treibhauseffekt und Ozonloch, Verwüstung und Verseuchung ganzer Landstriche, Ausrottung der Arten, atomarer GAU und Übervölkerung des Globus kein Thema. Äußere Restriktionen eines Prinzips, das aus sich vernünftig zu begründen war (und es immer noch ist), gab es nicht, jedenfalls nicht als »man-made « Grenzen. Aber kann heute das abstrakte Prinzip eines Weltbürgers in Freiheit bemüht werden, ohne Gedanken über die Restriktionen seiner konkret-historischen Realisierbarkeit oder über die »Antiquiertheit des Menschen « in einer entmoralisierten Systemwelt technisch-wissenschaftlicher und ökonomischer »Sachzwänge « anzustrengen? Man kann es, aber um den Preis der Zerstörung des Prinzips der Weltbürgerschaft zu Gunsten eines anderen: desjenigen der globalen Apartheid, die in jeder einzelnen Gesellschaft ihre Metastasen der erzwungenen Distanzierung ausbildet. Völker, die sich nicht selbst ernähren können, so ganz unverschämt F.A. von Hayek, sollen eben zugrundegehen. Völker, die nicht zum Wohlstand westlicher Industrienationen aufschließen, so ein Trend in der postmodernen politischen Theorie von rechts bis post-links, sind selber schuld an ihrer Entwicklungsmisere. Die liberale Emphase der Weltbürgerschaft und der Aufruf an die 
Rechte und die Linke, sich am Kreuzzug gegen die fundamentalistische Bedrohung des aufgeklärten Projekts von Markt, Demokratic und Individualität zu beteiligen, sind die Kehrseiten der gleichen Medaille. Eben darum ist der Appell zum antifundamentalistischen Feldzug so obszön - und gleichzeitig nutzlos. Denn es sind diejenigen, die zum Appell blasen, die mit ihrem Lebensstil, ihrer Produktionsweise gerade die Grenzen des Wohlstandsmodells erzeugen, das zwar aller Welt das Bild der Entwicklung bietet, aber gleichzeitig dafür sorgt, daß das Bild niemals weltweit Realität werden kann.

\section{Europa und der Rest der Welt}

Kapitalismus ist eine Produktionsweise, eine Gesellschaftsform und auch ein Lebensstil. Die Internationalisierung des Kapitalismus bedeutet also auch die Internationalisierung einer Lebensweise, die als American way of life ein Etikett erhalten hat. Infolge der Internationalisierung des Kapitals jedoch ist es nicht mehr möglich, einen American way of life auf nationalstaatlicher Ebene zu realisieren. Die Internationalisierung von Geld und Kredit hat die nationale Souveränität der Wirtschaftspolitik eingeengt, wenn nicht gesprengt. Gleichzeitig erfordert die internationale Konkurrenz Anpassungen der Produktionstechnologien, Arbeitsbedingungen und Arbeitszeiten. Angesichts dieses Sach- verhalts scheitern linke Projekte, bricht sich gewerkschaftliche Politik an den »Sachzwängen《 der internationalen Konkurrenzfähigkeit. Nationale Politik ist in der Internationalisierungsfalle gefangen und kann nur noch auf internationale Prozesse reagieren. Die neokonservative Deregulierungswelle der späten $70 \mathrm{er}$ und 80er Jahre ist folglich nicht nur einem ideologischen Konstrukt geschuldet, sondern der über Märkte vermittelten Internationalisierung. Auch der monetäre Keynesianismus spricht diesen Sachverhalt an: Staatliche Steuerungsmöglichkeiten sind angesichts der realen Marktlogik zum Mißerfolg verdammt. Die (nationalen) Staaten beherrschen in der internationalen Währungskonkurrenz noch nicht einmal die (ökonomische) Grenze. Ein Staat ohne äuBere und innere Grenzen, dies hat schon Poulantzas herausgearbeitet, ist letztlich nicht souverän. Die Versuche, auf regionaler Ebene politische Kompetenz zurückzugewinnen (z.B. mit dem innerhalb der Grünen favorisierten Projekt des »Europa der Regionen «), tragen diesem Sachverhalt Rechnung, werfen aber zugleich neue Probleme auf: die Konkurrenz von Regionen und die Unmöglichkeit, auf regionaler Ebene nicht nur Wachstums- sondern auch Schrumpfungspakte zu schlieBen. Um letztere nämlich abzusichern (beim Niedergang der Stahlindustrie, des Schiffbaus etc.), müssen überregionale, nationale, in Europa sogar supranationale europäische 
Umverteilungsprozesse kalkuliert und gesteuert werden, die die politische Präsenz auf überregionaler Ebene erforderlich machen. Nach Maastricht und den Beschlüssen über eine Währungsunion ist dies aber noch schwieriger als zuvor. Denn sollten die monetär-ökonomischen Kriterien von Maastricht tatsächlich in Europa etabliert werden (was einigermaßen unwahrscheinlich ist), wäre der Spielraum für eine sozialreformerische Linke oder für die Gewerkschaften höchst eingeengt. Auch mit linker »Europhorie (vgl. PROKLA 75) und Europa als »Ersatzutopie« für den verlorenen Sozialismus ließe sich dieses Defizit nicht beheben.

So kommt es zu dem paradoxen Sachverhalt, daß Produktion und Konsumtion die nationalen Grenzen ökonomisch sprengen und supranationale Institutionen hervorgebracht haben, die die politische Internationalisierung zumindest in Westeuropa auf die Tagesordnung gesetzt haben, und daß gleichzeitig durch nationalistische und ethnische Anrufungen individualisierende Distanzen erzeugt werden. Wenn »Wohlstand für alle (Europäer) « nicht als individuell befriedigende Abgrenzung realisiert werden kann, dann tendiert die »europäische Bürgergesellschaft « zum Wohlstandschauvinismus, dessen Mauern das politische Einigungsprojekt diskreditieren. Auch die moderne Rechte ist heute europäisch. Aber sie will ein geschlossenes Europa und rigide de- finieren können, wer dazu gehört und wer nicht. Sie will Europa für sich und die anderen, die Nicht-Dazugehörigen in den Rest der Welt expedieren. Das »Europa der Regionen « ist daher weniger ein freundliches Projekt der kleinen Kreisläufe und des »small is beautiful « als eine wohlstandschauvinistische Abgrenzung, ein regionalistischer Protektionismus innerhalb des europäischen Protektionismus. Die Legha Norte in Italien ist da bereits weit vorgeprescht. Europa hört da auf, wo Afrika anfängt, und das ist irgendwie in der Nähe von Rom.

Auch ökologische Überlegungen führen zu der Erkenntnis, daß die Globalisierung des in den entwikkelten OECD-Ländern realisierten Wohlstands- und Industriemodells gar nicht möglich ist, daß also die marktgesellschaftliche Individualisierung ein Hollywood-Traum ist, $»$ a dream that money can't buy«. Vordergruindig betrachtet hat dies mit der Begrenztheit von Ressourcen zu tun und mit den Grenzen der Tragfähigkeit der biotischen und abiotischen Sphären der Erde für die festen, flüssigen und gasförmigen Emissionen auch der »saubersten « Industrien. Jedoch dürfen diese Grenzen nicht als physische mißverstanden werden. Es handelt sich dabei um soziale Grenzen der Industrialisierung. Denn Industrialisierung ist eine Art »positionelles Gut" (Fred Hirsch). Dies bedeutet, daß die Gratifikationen der Industrialisierung nur genutzt werden können, 
sofern nicht alle Menschen dieser Erde daran partizipieren. Es ist schlechterdings ausgeschlossen, daß die mehr als sechs Milliarden Menschen am Ende dieses Jahrhunderts Fortschritt als eine der westeuropäischen oder nordamerikanischen vergleichbare Automobil- oder Kühlschrankdichte realisieren. Wenn alle das positionelle Gut industrielle Wohlstandsgesellschaft haben und nutzen wollen, hat niemand etwas davon. Die Produktion von Werten folgt einer Logik der Grenzenlosigkeit, jener der von Aristoteles beschriebenen »chrematistischen« Spirale«. Die Produktion und Konsumtion von Gebrauchswerten jedoch findet ein Maß an den menschlichen Bedürfnissen und an den natürlichen Grenzen der Lebenssphären des Globus, die als Umweltschäden im politischen Prozeß thematisiert werden und Resonanz in ökologischen Bewegungen finden.

\section{Selbstbegrenzung}

Fortschritt ist heute nur noch in der Sackgasse möglich, deren totes Ende jedoch bereits in Sichtweite ist. Ein linkes Projekt kann heute folglich nur noch postindustriell - und postfordistisch sein. Eine Fortsetzung der tradierten kapitalistischmarktwirtschaftlichen Industriegesellschaft ist nur scheinbar noch Fortschritt; das »Ende der Geschichte« könnte Ouvertuire zu den »letzten Tagen der Menschheit « sein. Denn erstens ist es völlig ausge- schlossen, daß alle Menschen dieses Modell zu praktizieren vermögen. »Internationale Solidarität«, also einer der zentralen Werte der Linken, würde ein niemals einzulösendes Schlagwort in einer Welt bleiben, in der Wohlstandsinseln sich nur halten können, wenn sie sich gegen die Zumutung der Ausdehnung des Wohlstandsmodells auf alle Kontinente zur Wehr setzen. Zum anderen ist schon heute absehbar, daß der Fortschritt am toten Ende der Sackgasse in eine heillose Flucht zurück umschlägt. Und dann ist gegen die regressiven Kräfte kein Halten mehr.

Ist Fortschritt also nur noch jenseits der Industriegesellschaft möglich? Auch ein linkes Projekt kann natürlich nicht auf moderne Technologien oder Industrien verzichten. Die industrielle Basis des Gesellschaftsmodells der Zunkunft ist unverzichtbar, jedoch muß diese der gesamten Menschheit zur Verfügung stehen, darf also kein positionelles Gut mehr sein. Die Richtung, die eine europäische Linke einschlagen muß, ist daher vorgezeichnet: Während die traditionelle Linke ihre Strategien zu beträchtlichem Teil auf Umverteilung gründete, kann eine moderne Linke ihr politisches Projekt nur noch als Strategie der Selbstbegrenzung, der Vermeidung, der Reduktion formulieren. Umverteilung setzt ja die Produktion des zu Verteilenden voraus, und daher hatte die Linke die geringsten Probleme, solange die Produktion expandierte, 
das Wachstum florierte und Vollbeschäftigung gesichert war. Die Ansätze, mit keynesianischer Stimulierung der Nachfrage das »golden age « der europäischen Wirtschaftswunder zurückzuholen, sind nicht nur problematisch, weil sie nicht funktionieren; sie wären noch problematischer, wenn sie funktionieren sollten.

Selbstbegrenzung, Vermeidung und Reduktion bedeuten zunächst eine radikale Verkürzung der Arbeitszeit, um die Beschäftigung zu steigern. Wie sehr solche Überlegungen dem herrschenden Trend zuwiderlaufen, zeigt die derzeitige Debatte um deren Verlängerung. Krisenzeiten sind immer Hochzeiten für populistische Großreden und Attacken, wenn politische Akteure am Ende ihrer konventionellen Weisheit angelangt sind, keine neuen Ideen wagen und Macht dort situieren, wo sie gerade sitzen. Das ist in Kiel nicht anders als in München oder Bonn (und Milano und Marseille). Wenn freilich alle versuchen sollten, Arbeitskosten durch Arbeitszeitverlängerung zu sparen und so die Konkurrenzposition zu verbessern, dann arbeiten schließlich alle mehr, aber keiner gewinnt im härteren internationalen Wettbewerb. Nur die Lebensqualität hat sich verschlechtert und die Arbeitslosigkeit ist nicht geringer, sondern größer geworden. Die $\gg$ Mitte der Gesellschaft «, aus der Nationalismus und Wohlstandschauvinismus entspringen, wird breiter. Die Strategie der Arbeitszeitverlänge- rung ist ökonomisch unsinnig und politisch kontraproduktiv. Denn man kann nicht den Stand der Emanzipation, wenn es denn in Krisenzeiten sich aufdrängt, nach hinten abwickeln, ohne politische Artikulationsmuster $\mathrm{zu}$ erzeugen, die auch nach hinten losgehen. Arbeitszeitverkürzung als »Suche nach der gewonnen Zeit $\ll$ bedeutet aber auch eine Abkehr von der tradierten Zentralität der Arbeit, also nicht nur neue ökonomische Strategien sondern ein neues kulturelles Projekt.

Darüber hinaus müssen radikale energie- und rohstoffsparende Methoden entwickelt werden. Auch hierbei handelt es sich nicht allein um intelligente technische Methoden, sondern um die Entwicklung neuer, mobiler und flexibler Konzepte und Lebensformen, um eine andere $\mathrm{Ge}$ staltung urbaner Verhältnisse, also auch um die Veränderung der Arbeitsteilung zwischen Stadt und Land, die Veränderung der Arbeitsteilung zwischen den Geschlechtern und derjenigen von körperlicher und geistiger Arbeit. Die Minimierung des ökologischen »Throughput «, des Stoff- und Energieverbrauchs, ist also mehr als Effizienzsteigerung, es zielt auf gesellschaftliche Verändeungen, die aus der Sackgasse der »modernen Industriegesellschaften« herausführen. Die ökologische Linke kann ein solches Projekt nur europaweit, international konzipieren. Auf die Herausforderungen nach dem Zusammenbruch des realen Sozialismus und der bipolaren Ord- 
nung ist mit einer »neuen Weltordnung « geantwortet worden. Sie wäre sozusagen die Umsetzung des historischen Konzepts vom »Ende der Geschichte in die (global)räumliche Dimension. Die politische und ökonomische Gestaltung der westlichen Industrieländer lieferten die Prinzipien dieser Ordnung. Nur sind deren Elemente nicht verallgemeinerbar. Die Kehrseite der schönen neuen Weltordnung ist das Chaos. die Unordnung. Die Aussichten sind also höchst unerfreulich: Krisen, Konflikte und Kriege in Permanenz. Sie lassen sich nicht auf Dauer von der »ordentlichen « Welthälfte fernhalten, zumal ja die »Ordnungsmächte « in den Regionen, wo das Chaos herrscht, ebenso permanent intervenieren, am Golf, am Horn von Afrika, in Jugoslawien, demnächst im Kaukasus und dann... Ein linkes Projekt muß ebenfalls universell sein, also vermeiden, den regressiven Tendenzen der ethnisch begründeten Abgrenzung mit der »nationalen Frage « nachzulaufen. Es muß zugleich multipolar konzipiert sein und den glaubwürdigen Versuch machen, die Polarisierung der gegenwärtigen Welt innerhalb der einzelnen Nationen, zwischen den Nationen und zwischen Norden und Süden auf dem Globus zu überwinden. 